\title{
La connexion
}

\section{implant-prothèse}

\section{The implant-prosthesis connection}

\section{MOTS-CLEFS : \\ - Connexion, implant, prothèse. \\ KEYWORDS: \\ - Connection, implant, prothesis.}

AOS 2013;264:9-13

DOI: $10.1051 / \mathrm{aos} / 2013403$

(C) EDP Sciences 2013

\section{Résumé}

Le succès de nos traitements implantaires à long terme, d'un point de vue biomécanique et d'un point de vue esthétique, dépend de la qualité de l'acte chirurgical implantaire et de la qualité de la réalisation prothétique. Ce dernier critère dépend de la connexion implantprothèse. L'orientation actuelle se fait vers la connexion interne, plus résistante, plus facile à manipuler, le cône-morse semblant présenter moins de micro-hiatus, moins de micromouvement. La connexion supracrestale limite la cratérisation et respecte l'espace biologique.

Le choix du système utilisé doit permettre la maintenance ò moyen et long terme, et donc être le plus universel possible.

\section{Abstract}

The long-term success of our implant treatments, from both a biomechanical and an aesthetic point of view, depends on the quality of the implantation surgery and of the prosthetics. The latter criterion depends on the implant-prosthesis connection. The current trend is towards an internal connection, which is more resistant and easier to handle. The Morse taper seems to present less of a micro-gap and less micromovement, because the supracrestal connection limits crater formation and preserves the biological space.

The choice of which system to use must ensure medium and long-term maintenance, and so must be as universal as possible.

Dr Jean-Luc Guillaume
diplômé PARIS V
DU de gérodontologie, DU de chirurgie buccale, DU de chirurgie osseuse implantaire,
DU de génie mécanique en implantologie orale
18, avenue Champleroy 89000 Auxerre

\section{INTRODUCTION}

Si l'on considère le nombre élevé de types de connexions, de formes de cols implantaires et de piliers proposés par les fabricants, les difficultés cliniques liées à la réhabilitation prothétique sur implant restent d'un niveau élevé, notamment par la cratérisation initiale et la péri-implantite [1] (fig. 1, 2 et 3).

La liaison implant-prothèse demeure un facteur déterminant de la durée de vie des traitements prothétiques sur implant [2].

\section{Connexion implantaire}

Elle assure la jonction implant-pilier prothétique. On distingue deux types de connexions, la connexion externe hexagonale et la connexion interne.

\section{Connexion externe hexagonale}

C’est la plus ancienne (fig. 4) (implant Bränemark), la partie mâle se situe sur l'implant, la partie femelle sur le pilier prothétique. La vis en or ou en titane assure la stabilité par serrage. Le logement est à l'intérieur de 


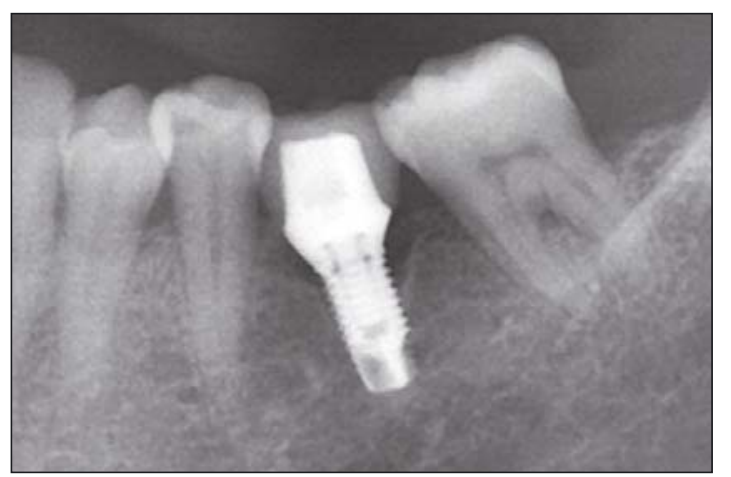

4 Fig. 1 :

Cratérisation péri-implantaire.

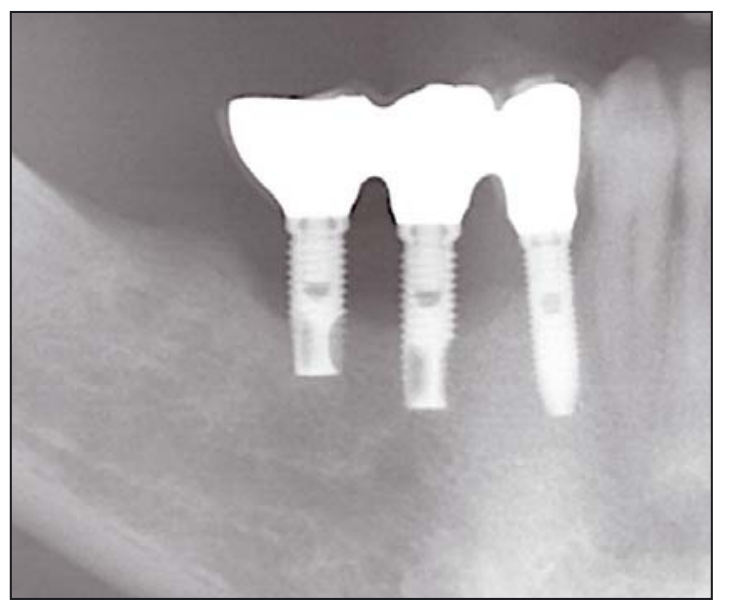

4 Fig. 2 :

Péri-implantite.

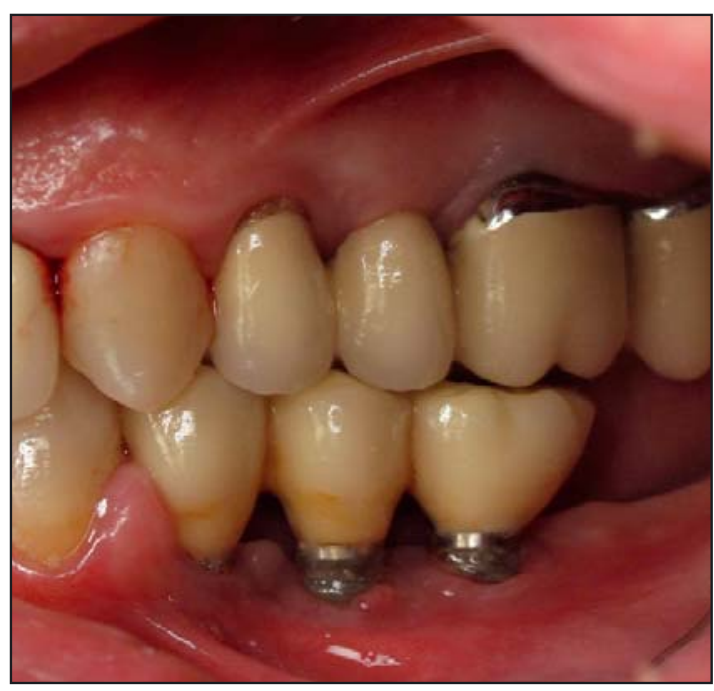

4 Fig. 3 :

Péri-implantite. l'implant. On a un système de connexion passif avec l'hexagone du col implantaire qui assure la fonction antirotationnelle.

Lautre connexion externe plus récente est la connexion spline de Zimmer. Elle est constituée de six créneaux d'un millimètre de hauteur avec un chanfrein au bord coronal des créneaux. Sa configuration facilite la manipulation, la précision et la simplicité. Elle limite les contraintes sur la vis et sur le col implantaire. Il n'y a pas d'incertitude sur le positionnement du pilier [3].

\section{Connexion interne}

La partie femelle se situe au niveau de l'implant, la partie mâle au niveau du pilier prothétique. Ce type de connexion fragilisant le col implantaire, il y a nécessité à utiliser des alliages de titane de grade 4 ou de grade 5 , qui présentent de meilleures propriétés mécaniques. On répertorie deux types de connexions internes :

I la connexion cône morse avec hexagone (fig. 5), c'est une connexion active. Le pilier est inséré en force dans l'implant, on obtient une pseudo-soudure à froid, même si la vis se desserre, le pilier reste en place. Cette connexion est précise, stable (antirotationnelle), on peut faire le reproche à cette connexion de ne pas maîtriser parfaitement l'insertion verticale ;

I la connexion à hexagone interne : elle est passive, c'est la vis qui tient le tout.

Des produits dérivés sont apparus : connexions polygonales, trilobées.

Ces différentes connexions internes donnent une meilleure sensation tactile. Elles sont plus résistantes, les micromouvements sont moindres ainsi le joint implant-pilier est de meilleure qualité, la vis est mieux préservée.

Entre connexion externe et interne, il était moins grave d'avoir une fracture de vis avec la connexion externe, que d'avoir à gérer une fracture de col implantaire avec la connexion interne.

Lutilisation de titane de grade 4 ou 5 évite, aujourd'hui, ce dernier phénomène.

\section{CRITÈRES BIOMÉCANIQUES}

1 Résistance à la flexion.

- Résistance à la fatigue.

1 Limiter les micromouvements.

1 Antirotationnelle.

\ Précision : qualité d'usinage.

1 Permettre un bon engagement du tournevis dans la vis par friction (cela évite la chute de la vis dans la cavité buccale).

I Positionnement précis (évitant la prise de cliché radiologique pour vérifier le bon emboîtement des pièces).

I Permettre une prise d'empreinte facile et exacte.

\Microgap le plus réduit possible (encore appelé 
micro-hiatus entre pilier et implant).

\ Le choix du matériau constituant le pilier prothétique influence la résistance mécanique :

Le pilier en alliage dor présente une bonne adaptation mais de moins bonne qualité mécanique. Le pilier en titane présente une bonne qualité mécanique et une bonne adaptation [4].

Le pilier en oxyde de zirconium a de grandes qualités mécaniques mais il y a un risque d'usure par micromouvements de l'implant.

Le pilier en oxyde de zirconium et titane, où la connexion se fait titane contre titane, présente de bonnes propriétés mécaniques et évite le phénomène d'usure par micromouvements [5].

\section{CRITÈRES BIOLOGIQUES}

Biocompatibilité du pilier implantaire $[6,7]$

Il semble que les piliers en titane et en zircone donnent de meilleurs résultats histologiques que les piliers en or et en platine. Les auteurs d'une revue de littérature concluent que le titane est le seul matériau qui présente une biocompatibilité avec les tissus mous.

Les autres matériaux sont moins biocompatibles [8]. Le micrograp permet la colonisation bactérienne du filetage interne de l'implant. Cela peut aboutir à l'installation d'une péri-implantite voire d'une lyse osseuse. D’après l'étude de Salihoglu et al. [9] où est comparée l'adhésion bactérienne de deux pathogènes parodontaux sur des piliers en oxyde de zirconium ou sur des piliers en alliage de titane, on ne note pas de différence significative.

D’après l'étude de Pappalasdo et al., 2007, le microgap augmente avec le diamètre de l'implant.

Létude de Dihart et al. [10] conclut qu'il n'y a pas d'infiltration bactérienne de l'intérieur vers l'extérieur et inversement avec la connexion conique.

Létude de Aloïse et al. [11] conclut le contraire. Létude de Rimondini et al. [12], indique qu'une pénétration bactérienne est possible dès lors que la connexion implant-pièce prothétique laisse paraître un microgap de 10 microns.

Létude de Cabratosa Termes sur les connexions coniques des implants Euroteknika montre qu'aucun microgap n'a pu être décelé à l'échelle du micron et qu'aucun assemblage pilier-pièce prothétique n’a laissé pénétrer de fluide à l'intérieur de la connexion.

\section{Caractérisation}

La caractérisation a poussé les industriels à chercher des designs implantaires pour supprimer ou réduire le problème.

Lespace biologique a une mémoire dimensionnelle [13]. En cas d'agression, il cherche à conserver ses dimensions, d'où l'apparition d'une cratérisation à la jonction implant-pilier, le tout favorisé par l'existence d'un microgap, puis par des micromouvements à l'interface pilier-implant après la mise en charge [14]. Abrahamsson et al. [15] montrent que le vissage-dévissage du pilier, s'il est unique, n'induit pas de remodelage de l'os marginal.

En revanche, la répétition de vissage-dévissage sur l'implant induit une résorption osseuse marginale.

Afin de stabiliser les tissus péri-implantaires, deux solutions sont proposées par les fabricants :

I favoriser la stabilité de la connexion implant-pilier avec l'utilisation d'une connexion interne cône morse ;

, éloigner la perturbation :

- par l'utilisation d'implant transgingival où la connexion est supragingivale (fig.6 à 10);
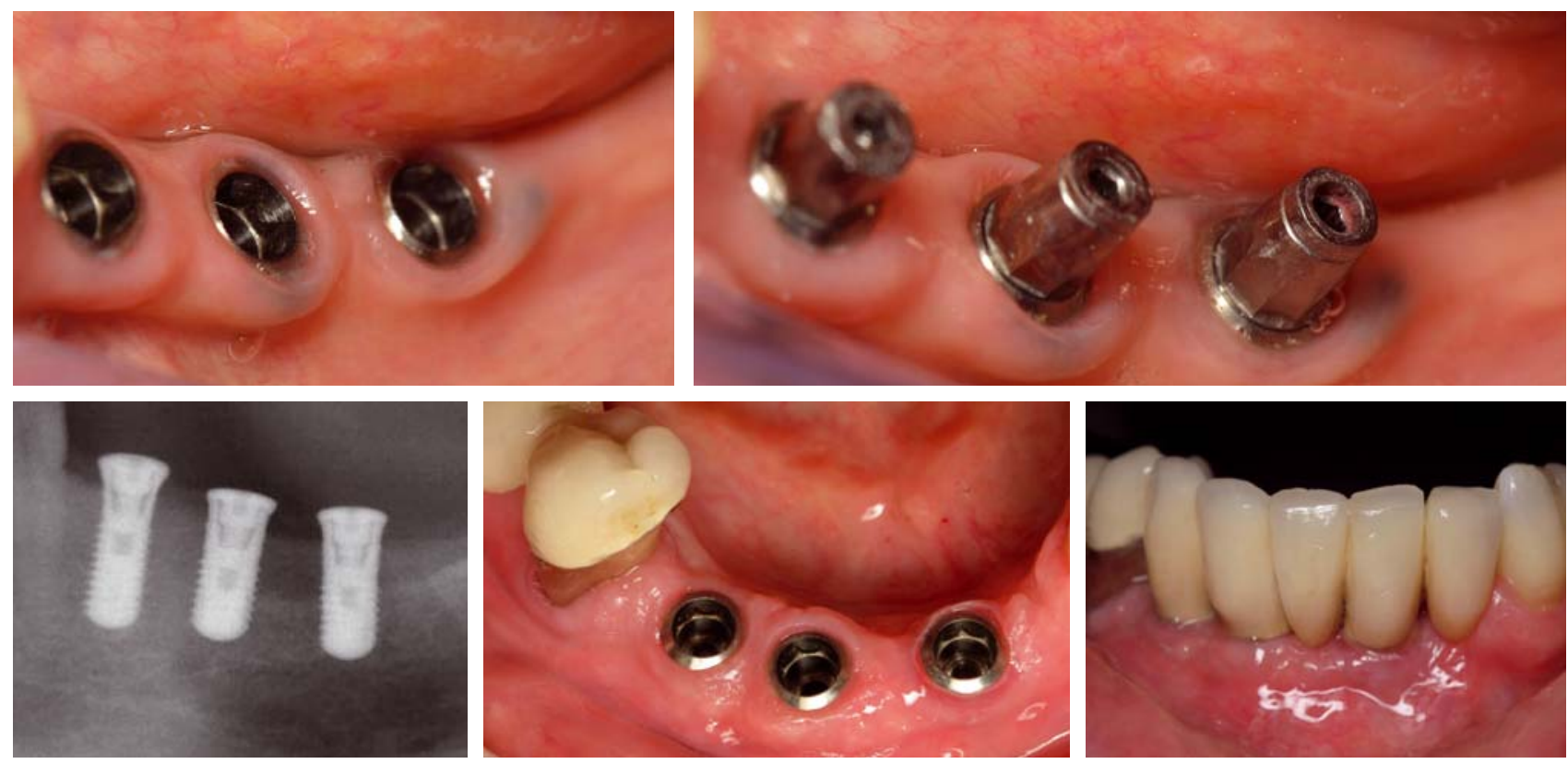

$\triangle$ Fig. 6, 7, 8, 9, 10 :

Connexion supra gingivale 


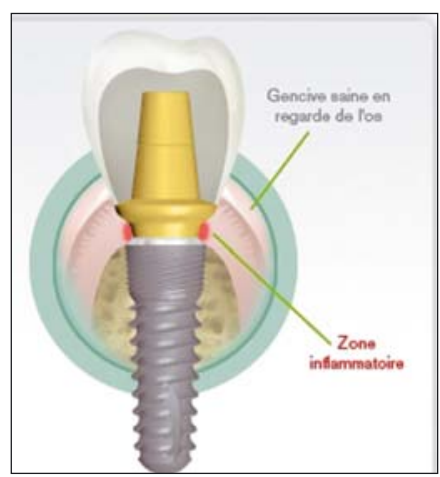

$\triangle$ Fig. 11 :

Platformswitching.
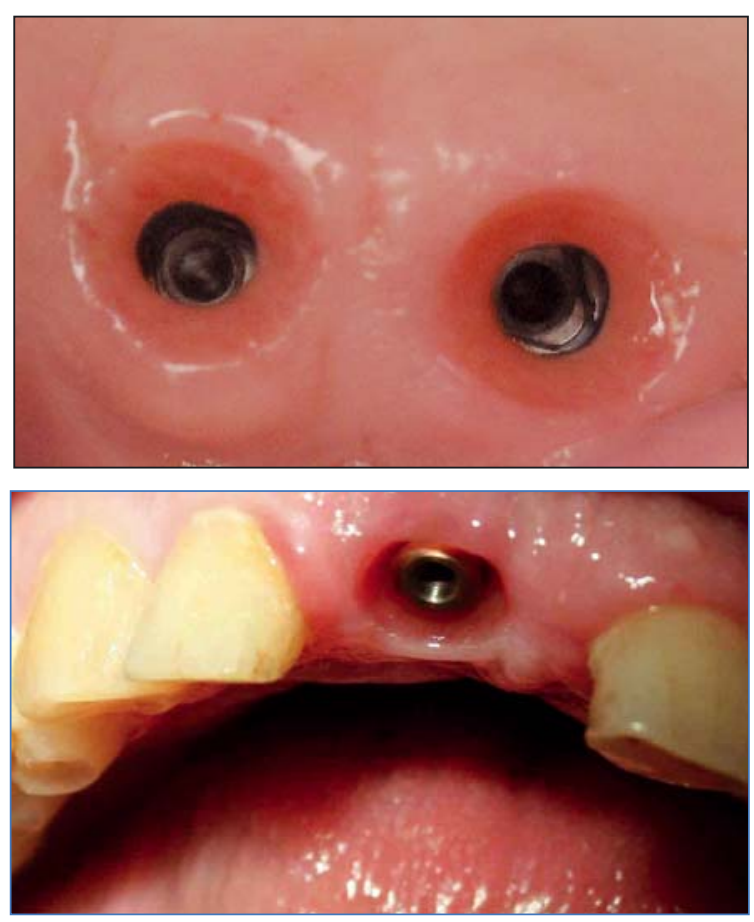

- par l'utilisation d'un pilier de diamètre inférieur à celui du col implantaire, c'est le principe du Platform-switching (fig. 11 à 13).

La jonction pilier-implant séloigne ainsi de l'os crestal péri-implantaire et met à distance l'inflammation due aux micromouvements [16-18].

\section{CRITÈRES ESTHÉTIQUES}

La première étape est le bon positionnement de l'implant, dans le sens vertical et dans le sens horizontal.

Il faut prendre en compte le diamètre de l'implant par rapport au diamètre cervical de la couronne, et les caractéristiques des tissus mous (classification de Meynard et Wilson). Le type de connexion influence l'espace vertical compris entre la plateforme de l'implant et les tissus mous marginaux. Avec une connexion externe, la hauteur de l'hexagone limite la place disponible pour utiliser un pilier implantaire et une couronne scellée.

Avec une connexion interne, on dispose de plus de place et on préférera ce type de connexion dans le secteur antérieur (Hanisch) (fig. 14).

En présence d'un parodonte fin, on utilisera un pilier en zircone, car un pilier en titane pourra créer une coloration grise de la muqueuse marginale.

Cependant, l'extrême dureté du zircone peut entraîner, en présence de micromouvements, une usure du titane implantaire [19].

Pour y remédier, certains fabricants proposent des piliers en deux parties : une base titane au contact de l'implant et une deuxième partie en zircone.

\section{Bibliographie}

[1] Theryl L. La connexion implantaire interne ou externe? L'Information Dentaire, 21 juin 2006, $\mathrm{n}^{\circ} 25$.

[2] Noharet R, Cannas B, Gillot L. Piliers implantaires : anticiper le choix. L'Information Dentaire, 19 mars 2010, $\mathrm{n}^{\circ} 20$.

[3] Schittly Jr, Russel P. Les implants Spline ${ }^{\circ}$. Chirurgie et prothèse. Editions CdP, 2002.

[4] Abrahamsson I, Cardaropoli G. Peri-implant hard and soft tissue integration to dental implants made of titanium and gold. Clin Oral Implants Res 2007;18:269-274.

[5] Seetoh YL. Load fatigue performance of conical implant-abutment connections. Int J Oral Maxillofac Implant 2011;26(4):797-806.

[6] Welander M, Abrahamsson I, Berglundh T. The mucosal barrier at implant abutments of different materials. Clin Oral Implants Res 2008;19(7):635-41.

[7] Abrahamsson I, Berglundh T, Wennström J, Lindhe J. The peri implant hard and soft tissues at different implant systems. A comparative study in the dog. Clin Oral Implant Res 1996;7:212-219

[8] Rompens E, Domken O, Degidi M, Pontes AEF, Piattelli A. The effect of material characteristics, of surface topography and of implant components and connections on soft tissue integration: a literature review. Clin Oral Implants Res 2006;17(suppl.2);55-67.
[9] Salihoglu U, Boynuegri D, Engin D, Duman AN, Gokalp P, Balos K. Bacterial Adhesion and colonization differences between zirconium oxide and titanium alloys : an in vivo human study. Int J Oral Maxillofac Implant 2011;26(1):101-107.

[10] Dibart S, Warbington M, Su MF, Skobe Z. In vitro evaluation of the implant-abutment bacterial seal : the locking taper system. Int J Oral Implant Maxillofac 2005;20(5):732-7.

[11] Aloise JP, Curcio R, Laporta MZ, Rossi L, Da Silva AM, Rapoport A. Microbial leakage through the implant-abutment interface of Morse taper implants in vitro. Clin Oral Implants Res 2010;21(3):328.35 Epub Janv 2010-2013.

[12] Rimondini L, Bruschi GB, Scipioni A, Carrassi A, Nicoli-Aldini N, 
Giavaresi G, et al. Tissue healing in implants immediately placed into postextraction sockets: a pilot study in a mini-pig model. Oral Surg Oral Med Oral Pathol Oral Radiol Endod 2005;100:43-50.

[13] De Sanetis M, Baldini N, Vignoletti F. Espace biologique péri-implantaire. JPIO 2011;29(4):245-259.

[14] Beauron E, Bousquet P, Torres JH, Gibert P. Les designs implantaires. Implant 2010;16:245-253.

[15] Abrahamsson I, Berglundh T, Sekino
S, Lindhe J. Tissue reactions to abutment schift: an experimental study in dog. Clin Implant Dent Related Res 2003;5:82-88.

[16] Jakubowicz-Kohen B, Rouach T, Rignon-Bret C. Esthétique et préservation tissulaire périimplantaire. L'Information Dentaire, juin $2008, \mathrm{n}^{\circ} 234$.

[17] Tabata LF, Rocha EP, Barao VA, Assunçao WG. Platform switching: Bio mechanical evaluation using Three-dimensional finite element analysis. Int J Oral Maxillofac Implant 2011;26(3):482-491.

[18] Bateli M, Wael Att, Strub Jörg R. Implant Neck configurations for preservation of marginal bone level: a systematic Review. Int J Oral Maxillofac Implant 2011;26(2):290303.

[19] Hanisch O. Piliers implantaires : critères de choix en vue de restaurations antérieures d'apparence naturelle. Stratégie Prothétique, septembre 2003;3(4).

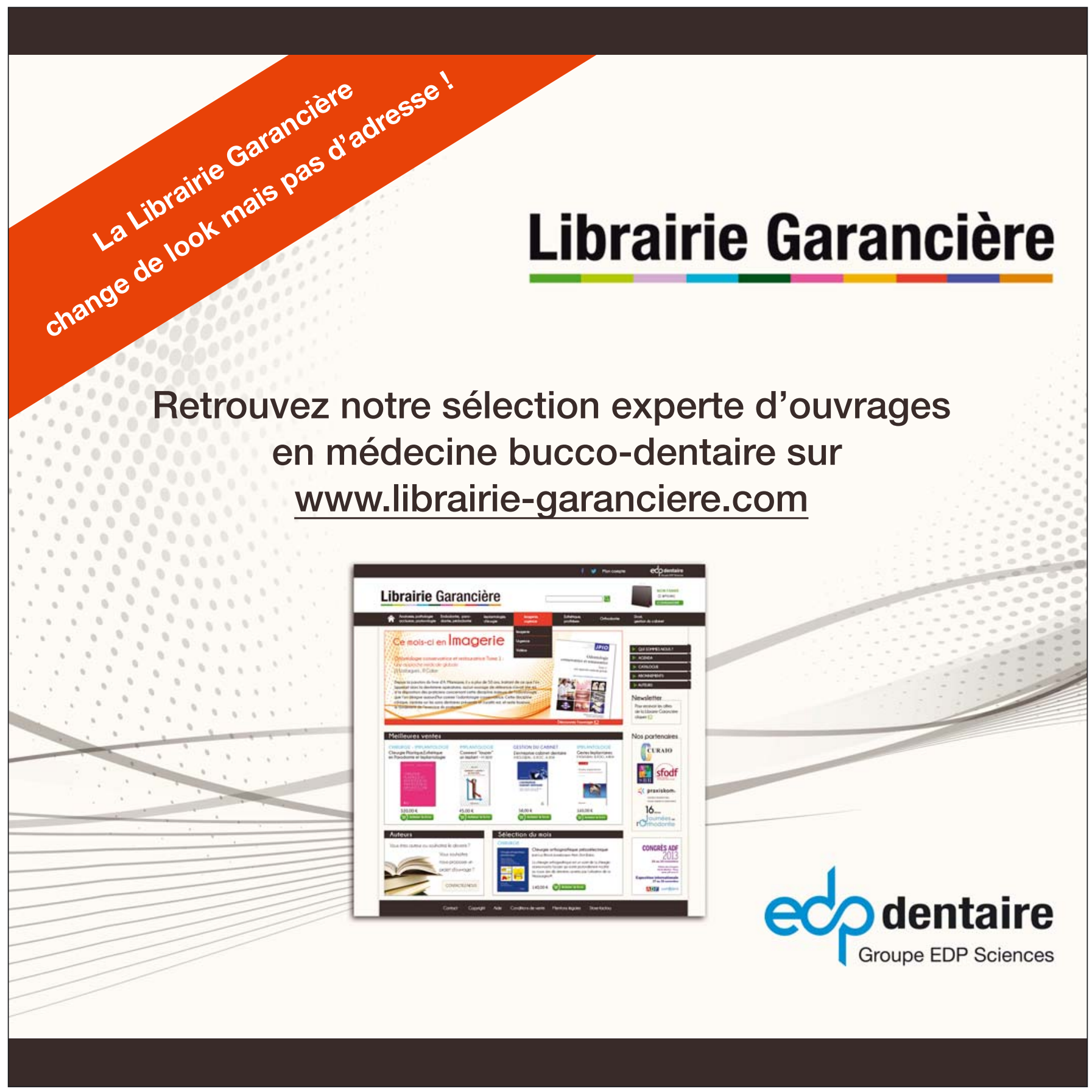

